Fecha de recepción: abril 2019

Fecha de aceptación: octubre 2019

Versión final: diciembre 2019

\section{Lo imaginario y lo maravilloso de Internet. Una aproximación antropológica}

Adriana A. Stagnaro *

Resumen: El objetivo de la presente contribución es interrogar a las temáticas presentadas en el film de Werner Herzog "Lo and Behold, Reveries of the Connected World" desde los aportes propuestos por la antropología de la ciencia y la tecnología. Aprovechando las palabras con las cuales comienza su comentario el crítico de cine Diego Maté (Cinemarama. Word Press, 5 de enero de 2017): "Hace tiempo que Werner Herzog oficia de antropólogo", podemos trazar una línea conectora entre los avances tecnológicos y la cultura dentro de la cual se producen.

La película trata sobre el surgimiento y desarrollo de Internet y de sus efectos en la cultura humana contemporánea. Para ello se vale de entrevistas a diferentes actores: ingenieros, astrónomos, matemáticos, emprendedores, integrantes de la comunidad científica vinculada al descubrimiento; y por otra parte a los legos que reciben los efectos de la interpelación tecno-científica en sus propios mundos privados. Los testimonios obtenidos dan cuenta de distintos enfoques, conceptos y percepciones configurando una mirada caleidoscópica, que oblitera cualquier interpretación simplista, obligando a la reflexión multidisciplinar.

En tal sentido, el objetivo es interpretar a partir de los distintos enfoques de la antropología de la ciencia y la tecnología, a las problemáticas relevadas en las respuestas atrayentes y brillantes de los entrevistados, logradas por cierto por la dialéctica herzogiana original y profunda. Así son puestas en tela de juicio las posiciones deterministas sobre la tecnología en las sociedades humanas, qué tipo de efectos y relaciones se establecen entre los nuevos descubrimientos y los valores sociales, culturales, políticos y éticos del mundo social. Nos preguntamos por el tipo de significaciones que la innovación científica-tecnológica conlleva y por otro lado, la capacidad de resignificación por parte de los sujetos.

Palabras clave: Internet, ciencia, cultura, sociedad, antropología

[Resúmenes en inglés y portugués en las páginas 71-72]

${ }^{(*)}$ Antropóloga. Doctora en Antropología Social por la Universidad de Buenos Aires. Profesora de la cátedra de Epistemología y Métodos de la Investigación Social en la Carrera de Ciencias Antropológicas de la Facultad de Filosofía y Letras y de Epistemología de las Ciencias Sociales en la Carrera de Trabajo Social, Facultad de Ciencias Sociales de la UBA. Como investigadora del Instituto de Ciencias Antropológicas de la Facultad de Filosofía y Letras fue una de las iniciadoras de la Antropología de la Ciencia y la Tecnología, especia- 
lidad sobre la que ha escrito artículos y capítulos de libros y sobre la que ha orientado su investigación, siendo su última publicación "Ciencia a pulmón. Etnografías de laboratorios argentinos de biotecnología”. 2015. Buenos Aires, Ciccus.

"Mis películas sólo son antropológicas porque tratan de explorar la condición humana en este momento particular del planeta. No hago películas con imágenes de nubes y árboles, trabajo con humanos porque me interesa la forma en que funcionan dentro de distintos grupos sociales". Werner Herzog

\section{Introducción}

En el documental Lo and Behold, Reveries of the Connected World estrenado en 2016 Herzog plantea sus reflexivas preguntas sobre nuestra cultura tecnocientífica, mientras despliega los diversos momentos del desarrollo y alcance de los hitos tecnológicos del Internet y la interpelación constante y en incremento al desenvolvimiento del mundo científico y de la vida cotidiana de los sujetos. Por eso, Lo and Behold, Reveries of the Connected World no es sólo un documental sobre la historia del Internet, ni sólo una investigación en torno a los avances de la revolución informática, ya sea desde un enfoque optimista o pesimista respecto de la tecnociencia. Esta película se presenta como una recopilación profunda y compacta de los discursos de los actores seleccionados y entrevistados, de los significados a ellos atribuidos, de los efectos maravillosos y transformadores, como también de los miedos y resquemores generados por una innovación tan pregnante y transversal de las prácticas y representaciones de científicos y legos.

En el comienzo Herzog reconstruye los momentos iniciales de Internet y para ello se traslada hasta la Universidad de California en Los Ángeles (UCLA) donde, en un resguardado espacio se presenta, cual extraordinaria obra de arte, una minicomputadora de grandes dimensiones y diseño antiguo, máquina donde tuvo lugar el 29 de octubre de 1969 la génesis de una de las revoluciones más importantes de la humanidad.

Su entrevistado y presentador, Leonard Kleinrock, uno de los pioneros en el desarrollo de Internet responde entre orgulloso y admirado las preguntas del cineasta: ¿¿ónde y cómo se inició Internet? ¿En qué lugar se creó la primera transmisión entre máquinas? ¿Cuál fue su primer mensaje? Con la ayuda del contexto sociotécnico e histórico brindado por un personaje central como lo es Kleinrock, Herzog presenta en su película la descripción de la primera computadora que emitió un mensaje. Esa mítica transmisión quedará grabada para la posteridad. Al querer transmitir LOG y esperar la respuesta IN (login), la máquina no logró transmitir la G, por lo cual el primer mensaje de todo el Internet fue LO, que da inspiración para el original título de la película. Lo and Behold es una expresión arcaica o humorística de la lengua inglesa que significa, literalmente, " He aquí!”, "Y quién lo iba a decir!" y que expresa sorpresa y admiración. Hay otras traducciones: "detente y admira", "observa y admira", "admira y sorpréndete" en relativa consonancia. Este va a ser el sentido que traspasa el resto de los diez capítulos que componen la película, un recorrido en 
el que a cada momento, al descubrir las oscuridades y las maravillas de Internet, Herzog nos invita a detenernos brevemente y admirar, sorprendidos, esta inabarcable creación humana, éste "sistema nervioso mundial" en palabras de su creador, que ha cambiado tan radicalmente, la cultura humana.

\section{Interconexiones históricas}

Según el enfoque relacional que sostiene que las audiencias están enroladas en la relación establecida entre los realizadores de películas sobre la ciencia y los científicos , participando las tres partes en la generación de nuevo conocimiento sobre el mundo natural y social (Gouyon, 2016), y como integrante del público y como antropóloga, sentí la necesidad de ampliar aquéllas preguntas básicas, a través del trabajo minucioso de un especialista sobre el tema como lo es Manuel Castells. En su ya clásica obra La Galaxia Internet publicada en el año 2001, nos presenta un estudio detallado de los antecedentes del surgimiento de Internet, desde la perspectiva de la tecnología y sociología de la sociedad de la información, que ayudan a desplegar la mirada histórica puntual ofrecida en el documental. Por supuesto que dicho despliegue además de aportar generoso conocimiento, suscita nuevas problemáticas e interrogaciones.

Castells $(2001$, p. 16) releva la coincidencia de tres procesos independientes dados en el último cuarto del siglo XX que encauzaron una nueva organización del mundo social basada predominantemente en las redes, antigua trama de lo social, ahora resignificada atendiendo a las necesidades de la globalización o mundialización del capital, la producción y el comercio, a las exigencias de flexibilidad en lo ateniente a la gestión; al valor atribuido a la libertad individual y a la comunicación abierta y finalmente a los progresos que experimentaron la informática y las telecomunicaciones, en base a los aportes de la revolución de la microelectrónica.

Con sus palabras:

En estas condiciones, Internet, una oscura tecnología que tenía poca aplicación más allá de los mundos aislados de los científicos informáticos, los "hackers" y las comunidades contraculturales, se convirtió en la palanca de la transición hacia una nueva forma de sociedad: la sociedad red -y con ella, hacia una nueva economía (Op. cit., p. 16).

Así es como Internet se erige como un medio de comunicación que habilita, por primera vez, la comunicación de muchos actores a una escala global. Y estableciendo una percibida analogía, Castells expresa: "Del mismo modo que la difusión de la imprenta en Occidente dio lugar a lo que McLuhan denominó la Galaxia Gutenberg, hemos entrado ahora en un nuevo mundo de la comunicación: la Galaxia Internet” (Op. Cit, p. 16).

Lo que magistralmente muestra Herzog en su documental de 2016 ya tenía sustento teórico en el Castells del 2001, donde sostenía que las principales actividades económicas, sociales, políticas y culturales de todo el planeta se están estructurando por medio de Internet. El film da cuenta de la velocidad de esa transformación en sus múltiples aspectos, 
pero sin pasar inadvertido el esfuerzo puesto por parte del documentalista en la tarea de seguirla en imágenes e interpretaciones, como asimismo le ha costado al mundo de la investigación académica, seguir analizando dicho fenómeno a la luz de investigaciones empíricas en todo el tiempo transcurrido. El relativo vacío de investigaciones fiables a principios del milenio ha sido hoy sustituido por un robusto corpus de investigaciones sociales provenientes del movimiento de los estudios sociales de la ciencia, punto de convergencia de análisis socioculturales y cognitivos desde nuevas ramas de la filosofía, sociología y antropología de la ciencia y la tecnología, estudios culturales y feministas (Fischer, 2007; Escobar, 1994; Latour, 2014; Downey\& Dumit, 1997; Stagnaro, 2002) que han tratado de superar las tensiones entre tradicionales miradas alentadoras y escépticas de los posibles efectos de Internet en la dinámica social.

En la introducción a su libro La Galaxia Internet, Castells (2001) propone una conceptualización no determinista de la tecnología y por ende, una comprensión de la red de redes como un modelo socio-técnico interactivo. En la medida que Internet va transformando la forma en que nos comunicamos y partiendo de la idea de que la actividad humana o cultura se basa en la comunicación vía el lenguaje, se desprende entonces que Internet va a interpelar necesariamente los distintos modos de organización sociocultural y en especial al mundo de la vida cotidiana, afectándolos profundamente. En la medida que los sujetos son interpelados constante y significativamente por esta nueva tecnología, generan nuevas prácticas e interpretaciones a partir de su uso, quebrando el efecto de "caja negra" de la tecnología.

Internet, planteada originalmente como una tecnología de libre comunicación por una serie de eventos históricos y culturales no es intrínsicamente liberadora, pero si una tecnología particularmente flexible, pasible de experimentar constantemente modificaciones a partir del uso social, del contexto y del proceso en los que se conforma. Por ello, afirma Castells, "Internet no es ni una utopía ni una distopia", es el medio por el cual hemos logrado comunicarnos, al que debemos analizar y comprender culturalmente si aspiramos a cambiar la realidad.

La confluencia de datos provistos por la historia de la ciencia y la tecnología y los datos sociales y culturales ayudan a entender la insólita fórmula dentro de la cual emerge Internet. Según Castells, los componentes de esa fórmula impensada fueron: la gran ciencia (big science), la investigación militar y la cultura libertaria en su acepción europea que refiere a una cultura o ideología cuyo principal valor radica en la libertad individual, según el pensamiento de John Stuart Mill.

Dentro de este contexto, el encuentro entre la investigación proveniente de las más importantes universidades y los asesores especializados en temas de defensa, fue esencial al desarrollo de esta tecnología. Así por ejemplo el proyecto tecnológico ARPANET fundado en 1962 como agencia de proyectos de investigación avanzada que se originó en el Departamento de Defensa de Estados Unidos para reclutar recursos de las universidades, con el objetivo de compensar la superioridad tecnológica alcanzada por la URSS en 1957, con el lanzamiento del primer SPUTNIK.

Castells desarrolla una especial historia social de Internet en búsqueda de "lecciones" a sacar de la evolución que va desde la creación de ARPANET hasta la explosión de la World Wide Web, una aplicación que permitió la mundialización de Internet desarrollada por 
Tim Bemers-Lee, un programador inglés en 1995, teniendo muy en cuenta que el surgimiento de una determinada tecnología en un lugar específico y en un momento histórico particular estipula tanto su contenido como los usos que se hacen de ella en su desarrollo futuro. Por ello, una visión panorámica de la historia de Internet interesa para comprender mejor su futuro progreso, que es el tema central de Herzog resumido en la interrogación metafórica final del film: “¿Se piensa Internet a sí misma?”

Por ese entonces el sueño era de los científicos y consistía en cambiar el mundo por medio de la posibilidad de comunicación entre computadoras. Bob Kahn y Vint Cerf generaron un protocolo de Internet que habilitó la explosión informática. La comunidad científica desplegó un rol principal en la creación de ARPANET, atrayendo a los doctorandos en las tareas de investigación y diseño de la red, favoreciendo un espacio de producción científica afable y dúctil, sin restricciones vinculadas a la seguridad. Y fue la National Science Foundation quien se encargó de viabilizar la transición hacia la privatización de Internet con la participación de la comunidad universitaria de informáticos que había desarrollado gran número de gente competente en aquel ambiente.

Según Castells (2001, p. 33) ARPANET, si bien nacido en cuna castrense -el Departamento de Defensa de los Estados Unidos-, no fue un proyecto de carácter esencialmente militar, aunque ese hecho tuvo efectivamente consecuencias en el desarrollo de Internet al momento de poder reunir los recursos humanos y materiales para erigir una red informática. El incremento de los fondos destinados al avance de la ciencia y de la tecnología, en el marco histórico de la guerra fría intentaba desafiar el palmario avance de la URSS en esas esferas demostrado con el éxito del proyecto espacial soviético, percibido como especialmente peligroso para la seguridad nacional estadounidense.

En ese contexto se inscribe la revolución de la microelectrónica y la emergencia de la innovación de Internet, lo que no constituye una excepción en la historia de las innovaciones tecnológicas. Lo que si fue insólito es que el Departamento de Defensa de los Estados Unidos dotara a ARPANET de una oficina de financiación y asesoramiento de la investigación (ARPA) con un alto grado de autonomía.

Kahn y Cerf quienes soñaban con desarrollar una red con capacidad para funcionar como un sistema abierto con alcance global alcanzaron a hacer sus sueños realidad, gracias a que gozaba de una relativa autonomía, que permitió que sus proyectos no fueran diseñados teniendo en cuenta puntuales intereses militares. Entonces esta condición abierta de la estructura de Internet constituyó su principal poderío y posterior ubicuidad.

\section{Actores interpelados por la ciencia y la tecnología}

En la parte II del film denominada "La gloria de la red", Herzog entrevista a científicos y tecnólogos de centros académicos de excelencia norteamericanos. Los testimonios revelan una fascinación positiva por las experiencias llevadas a cabo. Adrien Treuille científico en computación, involucrado en una investigación sobre SIDA y cáncer crea un videojuego y lo sube a la red para resolver un acertijo sobre el modo de configuración de diversas moléculas. Sorprendido, advierte que muy rápidamente distintos tipos de personas, científicos y legos, todos hábiles jugadores de videojuegos entraron y colapsaron a la web, hablaron 
entre sí, estudiaron y trataron de resolver el acertijo. Las soluciones fueron sometidas a verificación. No obstante, aclara el entrevistado defendiendo el principio de autoridad científica "es la naturaleza quien decide quien gana y quién pierde. Eso es ciencia". Otro ejemplo de participación activa en la red la comenta Sebastian Thrun, robotista y educador, conocido por el diseño de coches autoconducidos por computadoras. A fin de allegar el conocimiento a todos los alumnos que se inscribieran en la empresa que él creó, Udacity dedicada a la educación gratuita, pudo observar que muy velozmente se ampliaba el número de alumnos inscriptos, llegando al número de 160.000. Comparado con el de 200 que eran sus alumnos de Harvard, surgió que por cada buen estudiante de Harvard existían 413 mejores en el ranking pertenecientes a distintos lugares del mundo. En sus palabras, magnífica experiencia.

Estos hechos evidencian la capacidad de los sujetos denominados cyborgs por Dona Haraway (1985), es decir aquéllos interpelados por la tecnociencia, para participar, configurar y responder a ella en sus prácticas y discursos según sus intereses y experiencias humanas cotidianas. Según Downey y Dumit (1997), justamente en la frontera entre los cyborgs y los citadels, estos últimos habitantes de las ciudades fortificadas de la ciencia, la tecnología y la medicina, es donde se debe encontrar el antropólogo a fin de observar cómo la ciencia gana y mantiene la autoridad a través de prácticas de verdad y construcción del poder. Tarea esta que supera el enfoque ya algo naive de aquellos autores que resaltan la capacidad de los usuarios de producir y transformar la tecnología y aportar nuevas configuraciones y conectividades.

No obstante, por otro lado Internet, al trabajar con bancos de datos, íconos visuales, videoclips, películas, animación y cascadas de información remodela el viejo orden cultural apoyado en la oralidad y la alfabetización, lento en su producción y de formato rígido, reestructurando de esa forma la esfera pública a través del cambio en las relaciones de poder, como ocurrió en las relaciones médico-pacientes-empresas, movilizando fondos para campañas electorales o para la inversión en empresas biofarmacéuticas (Fischer, 2007, p. 37). Las transformaciones operadas en la tecnociencia y en la vida cultural por medio de la incorporación de masivas corrientes de datos, nuevas formas de visualización, nuevas formas de colaboración y una intensa comercialización, constituyen un área clave y un verdadero desafío para el análisis antropológico del mundo contemporáneo. Los nuevos ensamblados como Internet deben ser entendidos, para su mejor comprensión, como sistemas experimentales (Rheinberger, 1997, p. 2) donde confluyen al mismo tiempo los elementos locales, individuales, sociales, institucionales, técnicos, instrumentales y en especial los epistémicos constitutivos de los objetos científicos o tecnológicos bajo estudio. Son los lugares híbridos e impuros donde se generan las novedades tecno-científicas y sus significados, vehículos de incubación de preguntas, amalgamas de conceptos a medio camino, técnicas de no largo tiempo y los no todavía valores y estándares (Rheinberger, 1977, p. 36). Asimismo son lugares de coproducción de conocimientos. En algo más que una década -advierte Sheila Jassanof (2004)-, la amorfa entidad llamada Internet, cuya organización y gobierno sigue siendo un misterio para la mayoría de sus usuarios, llega a ser una actora en innumerables transacciones sociales. Al explorar sus posibilidades, millones de personas comienzan a alterar no solo la arquitectura de Internet, sino también sus propias preconcepciones de lo que significa pertenecer a unidades sociales 
como la familia, la comunidad, el lugar de trabajo, la empresa o la nación. La suma de sus interacciones ha cambiado las características del comercio, de las empresas, del capital, produciendo integración y disrupción en escalas globales. Por ejemplo, se puede afirmar que el diseño de tecnología es también casi accidental, flexible, inestable, porque refleja los imaginarios sociales, las preferencias y elecciones culturales y los recursos económicos o políticos, tanto de los innovadores, como de los usuarios (Bijker, 1997; Bijker et al., 1987). Y consecuentemente, que el enfoque disciplinario tradicional no está favoreciendo la comprensión de las intersecciones de la ciencia y la tecnología con lo político y la cultura, como ya desde hace 50 años lo está haciendo la investigación en estudios sociales de la ciencia y la tecnología. Muchos de estos investigadores han demostrado que la ciencia y la tecnología pueden ser estudiadas como prácticas sociales tendientes al establecimiento de distintos tipos de estructuras y jerarquías. Cada vez más la experiencia humana se presenta como un ensamblado de logros científicos, tecnológicos y sociales. En palabras de Jassanof (2004, p. 16): "en una palabra, son coproducidos, cada uno de ellos asegura la existencia de los otros".

En el film analizado, la mirada de su director se condice con esta postura, buscando en el reconocimiento de un nuevo fenómeno, analizar profundamente cómo los seres humanos organizan y reorganizan sus ideas de la realidad bajo determinadas circunstancias y los miles de posibles ensamblados entre las prácticas científicas y las sociales en tiempos de conflicto y cambio.

Para ello, Herzog se adentra en el corazón de la comunidad científica y tecnológica, en esa citadel o ciudad fortificada de la Ciencia y la Tecnología (Carnegie Mellon Institutes) a través de incisivas entrevistas a científicos, tecnólogos, informáticos, innovadores, empresarios de la ciencia, relevando con curiosidad compartida las categorías nodales del discurso científico: la búsqueda de lo nuevo, la capacidad de aplicar el conocimiento, la capacidad de transformar el mundo e interpelar a los sujetos.

Por otro lado analiza el discurso de los sujetos interpelados (Cyborgs) cómo estos reciben esas nuevas ideas y formas tecnológicas en la experiencia de sus vidas cotidianas. Aquí aflora cierta mirada pesimista: la actual dependencia de Internet, y la imposibilidad de aislarse de él. El retrato de una comunidad que se aísla voluntariamente de Internet parece un sueño idílico, pero encubre en realidad una reacción, cierta sospecha y el placer de lograr vivir de otro modo al impuesto. Si mañana desapareciera Internet por una explosión solar masiva, como ya se ha registrado en nuestro planeta ¿qué pasaría con el sistema de intercambio global? ¿Cómo se conseguirían los recursos para satisfacer las necesidades básicas como los alimentos, agua, fuentes de energía? ¿Qué pasaría con la ausencia de moneda y otros tipos de intercambio monetario y especialmente con el intercambio y el tejido social? Lo que aquí sugiere Herzog es que unos pocos podrían sobrevivir a ese colapso -los que ya hayan pagado su viaje a Marte- y que los prevenidos o aquellos que no confiaron ciegamente en la dependencia de satélites y computadoras van a heredar un mundo destruido por la necesidad de cierta clase dominante de obtener y acumular riquezas de todo tipo a cualquier precio que se deba pagar. Su visión del futuro es algo fatalista, lo que se muestra en la última escena de la película: el grupo de gente aislada de Internet, cantando felizmente música country, una reminiscencia y actualización del mito del buen salvaje. Los factores externos que rebasan nuestro control-como simplemente 
las constantes erupciones del sol-, pueden desestabilizar nuestro precario sistema sin que tengamos forma de detenerlo. Y la reflexión pesimista sobre nuestra dependencia de la conectividad da lugar a su vez a otros imaginarios tecnológicos. Si tenemos presente que nos podemos quedar sin planeta tierra, si agonizara nuestra civilización, ¿podremos encontrar nuevamente una esperanza en la tecnociencia? La citadel dice sí. Ya hay científicos y empresarios (Space X) pensando en instalar Internet en Marte para garantizar un hogar para aquéllos que quieran integrar colonias en posibles y viables planetas.

\section{Cómo analizar a la cultura Internet}

Más allá de los datos aportados en el film y el refinamiento y profundidad de su tratamiento en el contexto de las entrevistas y encuentros con distintos grupos registrados por Herzog, quedan para analizar ahora las formas en que la antropología de la ciencia ha estudiado y reflexionado sobre Internet.

Según Christine Hine (2015) el aporte original de la etnografía radica en ofrecer una comprensión basada en las percepciones humanas, generar thick data (datos densos) complementarios de los big data (datos fuertes). Esta mirada atenta a los actores permite contraponerse a la perspectiva que ya hace más de quince años define Internet como un mundo aparte, poblado de identidades virtuales. Esta concepción que pudo tener sentido en los comienzos de Internet ya no es más sostenible al momento de "web 2.0". Internet ya no es más un objeto único, sino, por el contrario, múltiple. Es un fenómeno cotidiano, integrado e incorporado (embedded) a las experiencias íntimas muy personales y a su vez, es un objeto universal que sobrepasa ampliamente la esfera privada. Por lo tanto se torna vano seguir buscando una ontología propiamente virtual. Según el punto de vista de Hine (2015, p. 104), que compartimos, es más pertinente pasar de una etnografía de Internet hacia una etnografía para Internet, que dé cuenta y aborde la multiplicidad de las experiencias localizadas en los puntos de contacto con el medio. En sus palabras: "un estudio de lo ordinario", es decir de las prácticas cotidianas. Es necesario captar que es lo que se trata en Internet, como los contenidos de los intercambios que se despliegan sobre las pantallas y también alrededor de ellas, es decir la inscripción de Internet en el seno de las prácticas cotidianas. La autora demuestra la capacidad de asociar ventajosamente el análisis de los contenidos en línea con la investigación de multisitios dirigida a seguir la movilidad de los actores (Marcus, 1995, p. 24, pp. 95-117) .También muestra con los resultados de las investigaciones de muchos casos concretos, cómo el proceso de enculturación del investigador es posible en el contexto del estudio de lo ordinario. Está convencida que la antropología dispone de todas las herramientas para interpretar los fenómenos sociales y culturales que se despliegan alrededor de Internet. Hine trata los desafíos contemporáneos del trabajo de campo que rebasan ampliamente al objeto Internet, como por ejemplo la incertidumbre, los múltiples sitios, las ontologías múltiples, las articulaciones entre tecnología y prácticas, la dificultad de acceder a los informantes menos activos, entre otros. Entonces, como orientación a los que quieran seguir su camino, Hine (2015) aspira a realizar una etnografía dinámica basada en un análisis en tiempo real de los fenómenos sociales, utilizando métodos móviles, el análisis de logs, de big data, la visualización de 
datos o el mapeo de las redes, recursos éstos que podrían enriquecer la mirada etnográfica no restringida a mirar de afuera o a intervenir en las márgenes de los fenómenos sociales. Desde otro enfoque, hemos seleccionado el trabajo que presentan tres jóvenes sociólogos franceses, Beuscart, Dagiral y Parasie (2016) donde se trata de responder a preguntas fundamentales que nos plantea la existencia de Internet: ¿ Cómo influye esta tecnología en la capacidad de acción de los individuos, en qué medida favorece las relaciones más simétricas o igualitarias, introduciendo mayor visibilidad de la propia sociedad? Para ello, toman como punto de partida la siguiente paradoja: por un lado Internet se desarrolla ilimitadamente en nuestras existencias cotidianas. Pero, por el otro, dados sus efectos sobre las formas de encontrarse, de discutir, de informarse, de consumir, de divertirse, de desplazarse, de intercambiar, de trabajar, etc. etc., éstos se constituyen en objeto de debates públicos. Si bien advierten que aún hoy la sociología no ha terminado de integrar a sus cuestionamientos y métodos el fenómeno Internet, es cierto que luego de los años 1990 se conforma un campo, de los estudios de Internet (Internet Studies) que no obstante se desarrolla al margen de los programas, temas de investigación y publicaciones de la sociología general. Si bien la sociología tributa a dicho campo junto con otras disciplinas, como los estudios de la comunicación y de los medios o de los estudios históricos y sociales de las técnicas y de la innovación, nunca termina por integrar a ese objeto inestable que es Internet.

Los autores (2016, p. 34), que tratan de demostrar la especificidad del nuevo campo de la Sociología de Internet, parten de una definición específica de ese objeto de estudio como un fenómenos de tres dimensiones dadas a la vez: la de las infraestructuras materiales y lógicas que permiten la comunicación entre máquinas conectadas; la de las prácticas diversas, de comunicación mediáticas o interpersonal, culturales, políticas; y finalmente la de las representaciones y discursos socioculturales. Tomando como punto de partida esta definición se preguntan en qué medida los principios, promesas y alcances del proyecto fundador de Internet influyen actualmente en las prácticas, tanto de los usuarios como de los proveedores de servicios. Así, el análisis de las comunidades que dieron origen a esas prácticas: los proyectos militares, las prácticas universitarias y la cultura informática permite reconocer tres indicios fundamentales. El primero, de índole social, da cuenta de la constitución de nuevas formas de sociabilidad en espacios inéditos, virtuales, que dan lugar a la conformación de grupos de interés independientes de la distancia geográfica y de la presencia física. El segundo, de tipo político, favorecería la conformación de una sociedad más democrática en virtud del efecto descentralizador e igualador de Internet al operar en el espacio público. La tercera, de carácter económico, genera una transformación de las relaciones de mercado y de los modos de producción, de comercialización y consumo de bienes.

A partir de estas notas, los autores seleccionan las investigaciones con el objetivo de que les permitan mostrar los cambios y rupturas, como también las continuidades y acumulaciones, sin caer en enfoques simétricos que reduzcan el efecto de Internet sobre la sociedad o por el contrario, lo sobrevalúen. Por ejemplo al examinar las promesas de mayor democracia en relación a las formas que tiene Internet de afectar el tratamiento de los asuntos públicos, subrayan que el aumento de la participación, la libre publicación y las nuevas formas de compromiso con lo público, se contraponen a la idea de que este fenómeno no es mecánico ni unívoco, sino por el contrario se presenta de forma limitada, desigual y dependiente de los medios de comunicación tradicionales. 
Igual conclusión sobreviene al análisis de los efectos de ampliación de las formas de acción colectivas y de su articulación con los medios de acción en línea y fuera de línea.

Si bien se comprueba una diversificación de las formas de compromiso y movilización política, no significa que ellas conduzcan automáticamente a una democracia participativa. Finalmente quiero resaltar que si bien la historia interna del surgimiento de Internet es a veces presentada como pura emergencia de acontecimientos técnicos y sociales muy entrelazados entre sí, no vinculados a ningún tipo de determinación económica o social, tal como aparece la innovación en sus primeros tiempos, considero que esta mirada contingente no se condice con la interpretación que ve en la evolución de los efectos Internet, ciertos límites y constricciones estructurales subyacentes que inhiben la inmediata democratización de todos los tipos de relaciones sociales afectadas. Si bien Internet opera como una cultura transversal (Shinn, p. 2002) que tiene la capacidad de atravesar varios mundos: el disciplinar-académico, el tecnológico, el empresarial, la vida cotidiana y el sentido común, es una configuración que mantiene sus tensiones y contradicciones sin poder desvanecer las persistentes relaciones de poder generadas por el conocimiento (Foucault, 1992) ni las de jerarquía y dominación (Bourdieu, 2003) producidas por lo social.

El film de Herzog habilita para plantear y profundizar el análisis de estos fenómenos que atraviesan la cotidianeidad actual. Siguiendo un movimiento detectado por los estudios sociales de la ciencia sobre la realización documental, ha sabido dar el paso desde una tecnología más al servicio de la ciencia a conformar un dispositivo reflexivo de ésta, con capacidad para cuestionar su autoridad y sus prácticas. Además de revelar la tensión encapsulada en cada obra entre lo artificial/entretenimiento y la evidencia/ciencia (Gouyon, 2016, p. 19), Lo and Behold interpela fuertemente a la audiencia poniendo a su disposición una serie de herramientas para reflexionar sobre los cambios en la cultura y la sociedad.

\section{Bibliografía}

Beuscart, J. S.; Dagiral, E.; Parasie, S. (2016). Sociologie d'Internet. Paris: Armand Colin.

Bijker, W. E; Hughes, T. y Pinch, T. J. (1997). “The Social Construction of Facts and Artifacts: Or How the Sociology of Science and the Sociology of Technology Might Benefit Each Other". En: The Social Construction of Technological Systems.Cambridge, The MIT Press. Bourdieu, P. (2003). El oficio de científico. Ciencia de la ciencia y reflexividad. Barcelona: Anagrama.

Castells, M. (2001). La Galaxia Internet. Barcelona: Plaza\&Janes Editora.

Downey Gary, L. y Dumit, J. (Ed) (1997). Cyborgs \& CITADELS. Anthropological Interventions in Emerging Sciences and Technologies. Santa Fe. New Mexico: School of American Research Press.

Escobar, A. (1994). "Welcome to Cyberia. Notes on the Anthropology of Cyberculture”. En: Current Anthropology. Volume 35, Number 3.

Fischer, M. (2007). "Four Genealogies for a Recombinant Anthropology of Science and Technology”. En: Cultural Anthropology. Volume 2. Issue 4. P 539-615.

Fischer, M. (2009). Anthropological Futures. Durham and London: Duke University Press. Foucault, M. (1992). Microfísica del Poder. Madrid: La Piqueta. 
Gouyon, J. B. (2016). "Science and film-making”. En: Public Understanding of Science Vol 25(I) $17-30$

Haraway, D. (1995). Ciencia, cyborgs y mujeres. La reinvención de la naturaleza. Madrid: Cátedra.

Hine, C. (2000). Virtual Ethnography. London: Sage.

Hine, C. (2015). Ethnography for the Internet. Embedded, Embodied and Everyday. London: Bloomsbury.

Jasanoff, S. (2004). States of Knowledge. New York: Routledge.

Latour, B. (2014). “Agency at the time of the Anthropocene”. En: New Literary History Vol. 45, pp. 1-18.

Marcus, G. (1995). "Ethnography in /of the World System: the Emergence of multi-Sited Ehnography”, Annual Review of Anthropology 24: 95-117.

Rheinberger, H. J. (1997). Toward a History of Epistemic Things. Stanford: Stanford University Press.

Shinn, T. and Joerges, B. (2002). "The transverse science and technology culture: dynamics and roles of research-technology". En: Social Science Information. London: Sage.

Stagnaro, A. (2002) “Tecnología y cultura humana” En: Encrucijadas. Revista de la Universidad de Buenos Aires. Año 2, Número 20:38-47.

\begin{abstract}
This contribution aims to interrogate the themes presented in the Werner Herzog film "Lo and Behold, Reveries of the Connected World" from the contributions proposed by the anthropology of science and technology. Taking advantage of the words with which begins his comments the critic of films Diego Maté (Cinemarama. Word Press, January $5^{\text {th }}, 2017$ ): "Long ago that Werner Herzog serves as anthropologist", we can draw a line connector between technological advancements and the culture within which they occur. The movie is about the emergence and development of the Internet and its effects on the contemporary human culture. For this purpose it relies on interviews with different actors: engineers, astronomers, mathematicians, entrepreneurs, members of the scientific community linked to the discovery; and on the other hand the legos that receive the effects of the technoscientific interpellation in their own private worlds. The obtained testimonies realize different approaches, concepts and perceptions by setting a kaleidoscopic look, that destroys any simplistic interpretation, forcing the multidisciplinary reflection. In this sense, the objective is to interpret from the different approaches of the anthropology of science and technology to problems relieved responses attractive and shiny interviewees, achieved by the way the dialectical herzogiana original and deep. So the deterministic positions on technology in human societies, what kind of effects are put into question and relations established between new discoveries and the social, cultural, political, and ethical values of the social world. We ask about the type of meanings inherent in technological innovation and on the other hand, capacity of resignification by subjects.
\end{abstract}

Keywords: Internet - science - culture - society - anthropology. 
Resumo: O objetivo deste trabalho é interrogar às temáticas apresentadas no filme de Werner Herzog Lo and Behold, Reveries of the Connected World desde os aportes propostos pela antropologia da ciência e a tecnologia. Aproveitando as palavras com as que começa seu comentário o crítico de cinema Diego Maté (Cinemarama.Word Press, 5 de janeiro de 2017): "Tem sido um tempo desde que Werner Herzog oficiou como antropólogo", podemos traçar uma linha de conexão entre os avanços tecnológicos e a cultura dentro da qual eles são produzidos.

O filme aborda o surgimento e desenvolvimento de Internet e seus efeitos na cultura humana contemporânea. Para isso faz entrevistas a diferentes atores: engenheiros, astrónomos, matemáticos, empreendedores, integrantes da comunidade científica vinculada ao descobrimento; e, por outra parte, aos leigos que recebem os efeitos da interpelação tecno-científica em seus próprios mundos privados. As testemunhas dão conta dos diferentes enfoques, conceitos e percepções configurando uma mirada caleidoscópica, que oblitera qualquer interpretação simplista, obrigando à reflexão multidisciplinar.

Neste sentido, o objetivo é interpretar a partir dos distintos enfoques da antropologia da ciência e a tecnologia, às problemáticas relevadas nas respostas sedutoras e brilhantes dos entrevistados, logradas pela dialética de Herzog original e profunda. Assim são postas em causa as posições deterministas sobre a tecnologia nas sociedades humanas, que tipo de efeitos e relações se estabelecem entre os novos descobrimentos e os valores sociais, culturais, políticos e éticos do mundo social. As perguntas que se fazem são o tipo de significações que a inovação científico-tecnológica traz e, por outro lado, a capacidade de ressignificação por parte dos sujeitos.

Palavras chave: Internet - ciência - cultura - sociedade - antropologia. 